\title{
The Effects of Cognitive and Motor Dual-Task Training on Improvement of Balance, Quality of Life, and Fear of Falls in People with Idiopathic Parkinson's Disease
}

\author{
${ }^{1}$ Tahereh Pourkhani*, ${ }^{1}$ Hassan Daneshmandi, ${ }^{1}$ Ali Asghar Norasteh, ${ }^{2}$ Babak Bakhshayesh \\ Eghbali, ${ }^{1}$ Parisa Sedaghati
}

${ }^{1}$ Department of Sport Science, Faculty of Physical Education and Sport Science, University of Guilan, Rasht, Iran. ${ }^{2}$ Neurology Research Center, School of Medicine, Guilan University of Medical Science, Rasht, Iran.

Submitted 20 August 2019; Accepted in final form 06 December 2019.

\begin{abstract}
Background. Parkinson's disease (PD) is characterized by motor and non-motor symptoms that affect patients' functional performances, especially while performing dual tasks which is a critical factor in connection with everyday living. There are many controversies about the benefits. Objectives. This study assessed the efficacy of motor and cognitive dual-task training programs for improving of balance, quality of life, and fear of falls in people with idiopathic Parkinson's disease. Methods. About 30 PD patients (Hoehn and Yahr stage II-III while ON medication) were assigned to a cognitive dual-task training group $(n=10)$, a motor dual-task training group $(n=10)$, or a single task control group $(n=10)$. All groups received 30 sessions of different exercises for ten consecutive weeks. Balance, quality of life, and fear of falls respectively assessed with Time Up and Go Test (TUG) and Trial Walk Test (TWT), Parkinson's Disease Questionnaire - 39 (PDQ - 39), and FES-I, before and after training and after one-month followup. Results. No significant time by group interaction were found, suggesting both dual-task and single-task training had a similar effect on outcome measures $(\mathrm{p}>0.05)$. A significant post-training improvement in $\mathrm{TUG}(\mathrm{F}=535.54, \mathrm{p}$ $<0.00)$, PDQ - 39 ( F = 463.52, p < 0.001), TWT $(\mathrm{F}=284.89, \mathrm{p}<0.001)$, and FES-I $(\mathrm{F}=266.4$, $\mathrm{p}<0.001)$ was found after single and dual-task training. These improvements were maintained at follow-up, although the effect was slightly reduced $(\mathrm{p}<0.05)$. Conclusion. Motor/cognitive dual-task training and single-task training were found to be moderately effective in improvement of balance, quality of life, and decrease of fear of falls in people with PD.
\end{abstract}

\section{KEY WORDS: Parkinson Disease, Dual-Task Training, Balance, Quality of Life, Fall}

\section{INTRODUCTION}

Parkinson's disease (PD) is the second most common neurodegenerative disorder after Alzheimer's disease that targets basal ganglia (1). It is estimated that there are 4 million people with PD in all over the world, and this number will double until 2030 (2). This illness is characterized by both motor and no motor symptoms that produce some challenges to activities of daily living and quality of life (1). The main motor symptoms include tremor, rigidity, bradykinesia, and postural instability (3). Patients with PD usually present with cognitive impairments including deficits in executive function, attention, working memory and visuospatial domains that can interfere with mobility (4). More than half of the people living with PD suffer from gait

*. Corresponding Author:

Tahereh Pourkhani, PhD

E-mail: zpourkhani@gmail.com 
disturbances according to the severity of disease (5) that causes falling so that between 50 to $68 \%$ of people with PD experience at least one fall during a year (6). In PD, reduced quality of life is associated with balance and gait abnormalities, including festination and freezing of gait (FOG) (7). Although medications such as levodopa are the primary treatment for PD, it becomes less effective for some symptoms after a long period of usage (5). Thus, the other kind of treatments like exercise therapy or physical therapy is needed for these patients.

On the other hand, dual-task (DT) performance refers to the ability to perform two tasks simultaneously at the same time. Mobility in daily life frequently requires DT performances such as talking and/or carrying a glass of water while walking. Several studies demonstrated some gait disturbances such as the increased risk of falling (8), more FOG (9), and reduced functional mobility (10) during DT conditions in patients with PD. According to the guidelines of physical therapy DT training is better avoided or used cautiously in PD (11) because it is not known whether it is a practical intervention. However, some recent studies showed the benefits of DT training in PD. Santos et al. in a systematic review, reported that different types of dual-task interventions could improve some gait parameters in patients with PD (3). As mentioned above, since the people in their activity of daily living face with DT condition a lot, therefore DT training could be challenging and beneficial specially in patients with balance disorders like PD. Another point is that, research examining the ability to modify DT performance among people with PD is very limited (12) and there is not clear indication for the use of DT, or of its effects as a therapeutic strategy in the literature. Therefore, this study investigates whether or not cognitive and motor DT training has any effect on balance, quality of life, and fear of falls in people with idiopathic PD.

\section{MATERIAL AND METHODS}

Design. The study protocol was approved by the Ethics Committee of Guilan University of Medical Sciences (No.IR.GUMS.REC.1396.381), recorded at the Iranian Registry of Clinical Trials (IRCT20180106038239N1) and conducted after obtaining the necessary permissions. A controlled clinical trial was conducted to compare cognitive and motor dual-task training with simple exercise.
The research period included ten weeks supervised training and one month follow-up and primary outcomes measured at baseline, after supervised practice, and after follow-up. In this study, patients were trained and assessed by a physiotherapist in a private clinic of physical therapy. On the other side, patients were tested on medications, at the same time of day for pre- and post-intervention and follow up assessments.

Participants. In this research, sample size estimation was performed by $G$ Power 3.1 software which is an excellent freeware program for sample size analysis (13-15). A total number of 30 subjects would be needed with $\alpha=0.05$, effect size $=0.5$ and review power equal to 0.8 . So, thirty patients with mild to moderate PD were recruited for the study. After evaluating eligibility, all patients were informed of the process of the research and signed informed written consent before the start of the study. Patients were allocated to a control group $(n=10)$ and two experimental groups $(\mathrm{n}=20)$ by quasirandom allocation method. In experimental groups, there were ten patients in the motor dualtask training group (MDTTG) and ten patients in the cognitive dual-task training group (CDTTG).

Inclusion Criteria. It was included:

1. Diagnosis of PD by a consultant neurologist, stage II-III on the Hoehn and Yahr scale (16),

2. Aged between 50 to 75 years old,

3. Stable medication regimen within the previous month and during the period of the research (4 months) (17),

4. Able to walk 100 meters independently without any assistive devices (5).

Exclusion Criteria. It was included:

1. Another neurological conditions in addition to PD such as CVA,

2. To have any musculoskeletal or cardiopulmonary conditions that affect the quality of life like surgery in hip or knee or severe DJD or RA,

3. Surgery for PD such as deep brain stimulation,

4. A score $<24$ in the Mini-Mental State Examination (MMSE),

5. Sensory impairment (e.g., blindness, deafness) (5),

6. Participation in an organized exercise therapy program in the last previous 6 months (17).

Outcome Measures. The outcome measures were the Timed Up \& Go (TUG) Test, Parkinson's Disease Questionnaire-39 (PDQ-39), Trail-Walking Test (TWT), and Falls Efficacy 
Scale-International (FES-I). TUG is a functional test in which the ability to rise from a seated position on a chair, walk $3 \mathrm{~m}$, turn, walk back, and sit down is timed. It is determined that the TUG test has a high test-retest reliability and interrater reliability in PD populations (18). Quality of life (QoL) is a universal concept that includes all dimensions of life. It has a physical, physiological, social and spiritual dimension. In chronic diseases, when treatment aims to increase the level of satisfaction and improve QoL in patients, the use of a standard instrument for measuring QoL is unavoidable (19). PDQ-39 is the first specific and invariable instrument for the assessment of health-related quality of life (HRQL) in PD which contains 39 items, including eight separate dimensions: mobility, activities of daily living, emotional well-being, stigma, social support, cognition, communication, and bodily discomfort. For each item, there is a range of five possible answers. The scores from each part are computed into a score ranging from 0 (best) to 100 (worst) (20). Dehghan et al. showed that the reliability and validity of the Persian version of PDQ-39 are acceptable. They reported that the range of Cronbach's alpha coefficient for different dimensions varied from 0.64 to 0.92 . Cronbach's alpha coefficient was higher than 0.70. Itemconvergent validity ranged from 0.38 to 0.83 . Thus, PDQ-39 can be used for measuring HRQL in Persian-speaking Parkinson's disease patients (21). Another test that was done in the current study was TWT. In the environment of TWT, flags are placed randomly at each of the 15 positions in a $25-\mathrm{m} 2$ area $(5 \times 5 \mathrm{~m})$. The participants are ordered to start moving from flag no. 1 to no. 15 subsequently, as quickly and correctly as possible. A $30-\mathrm{cm}$ diameter circle is drawn around each flag. The passage is considered to be successful when the participant stepped on the circle. The height of the flags is 30 $\mathrm{cm}$. The trial is timed using a stopwatch to the nearest $0.01 \mathrm{~s}$ and it is performed only once. Yamada and Ichihashi in 2010 reported that the test-retest reliability of TWT is high (interclass correlation coefficient 0.945 ). In their study, the stepwise logistic regression analysis revealed that the TWT was significantly related to falling in community-dwelling elderly individuals (22). The last outcome measure in the current study was FES-I. In 2005 the FES-I was developed to evaluate fear of falling while carrying out 16 tasks related to ADL. The total score varies between 16 (not worried) and 64 points (very worried). In a systematic review, Marques-Vieria et al. evaluated the reproducibility and validity of FESI. According to their results, the FES-I is acceptable for measuring fear of falling in the elderly and it is valid, reliable, and comparable cross-culturally. Thus, it is recommended in rehabilitation research and fall prevention program in an old population (23).

Intervention. In the single-task training control group (STTCG) and cognitive and motor dual-task training groups, patients participated in a 30-session program administered for 45 minutes each session, three times per week for ten weeks. A physiotherapist conducted the training in the ON medication condition (often 1 hour after medication). In the single-task group, patients made a selection of exercises presented by Parkinson's Society of Canada, including wall standing practice, tandem stance, single-leg stance, standing on toes, squat, march, side bending exercise, trunk rotation exercise, and figure of eight walkings (24). According to the overload principle of practice, the training program had a progressive trend. In the cognitive and motor dual-task groups, patients did the exercises while performing various additional cognitive or motor tasks. Other cognitive responsibilities during the training included counting backward by threes, memory recall, generating category lists (e.g., fruit, sports, names starting with a specific letter) and simple calculation tasks. Additional motor tasks were selected to reflect everyday activities such as doing up buttons, carrying a plate with a glass on top and transferring coins between pockets or objects like cell phones between hands while training (25). Patients were instructed to perform additional tasks while doing the exercises correctly.

Statistical Analysis. Statistical analysis was performed using SPSS version 20. Results obtained in the study were considered statistically significant at alpha value $\leq 0.05$. For assessing any differences between clinical and demographic variables at the beginning of the study, one way, ANOVA was used. In this study, four separate 2-factor [Time (Pre/Post/Followup) $\times$ Task (Single/Cognitive Dual)/Motor Dual] Repeated-Measures Analyses of Variance (RMANOVA) were used to establish the effect of time and task on primary outcome measures within each group. The greenhouse-geisser correction 
was used when the results obtained from the Mauchly's test of sphericity indicated a violation of the sphericity assumption $(\mathrm{p}<0.05)$. Bonferroni corrections were applied to multiple comparisons. Effect size (ES) was reported for all variables.

\section{RESULTS}

A total number of 32 patients participated to receive one of training protocols CDTT $(n=11)$, $\operatorname{MDTT}(n=11)$ or STT $(n=10)$. The dropout rate was $6.2 \%$ : one patient dropped out of CDTT, and one patient dropped out of MDTT because they could not finish the training protocols. The groups were similar in clinical and demographic variables. Table 1 shows that there are not any significant differences in the clinical and demographic variables of subjects at the beginning of the study using one way ANOVA ( $p$ $>0.05)$. All data are normally distributed using the Shapiro-Wilk test $(\mathrm{p}>0.05)$ therefore parametric statistic tests were used.

Table 1. Clinical and Demographical Variables of Subjects at Baseline

\begin{tabular}{|lccccc|}
\hline Variable & CDTTG & MDTTG & STTCG & F & p \\
Age, year & $67.2 \pm 3.79$ & $68.9 \pm 4.12$ & $67.9 \pm 3.78$ & 0.47 & $0.62^{*}$ \\
Duration of Disease, year & $5.8 \pm 1.93$ & $6.0 \pm 1.82$ & $6.6 \pm 2.06$ & 0.45 & $0.63^{*}$ \\
H \& Y, II-III & $2.8 \pm 0.42$ & $2.7 \pm 0.63$ & $2.65 \pm 0.57$ & 0.19 & $0.82^{*}$ \\
BMI, kg/m $\mathbf{2}^{2}$ & $23.60 \pm 1.13$ & $24.27 \pm 1.48$ & $23.20 \pm 0.79$ & 2.10 & $0.14^{*}$ \\
MMSE/30 & $27.70 \pm 1.05$ & $27.20 \pm 1.35$ & $27.60 \pm 1.17$ & 0.55 & $0.58^{*}$ \\
UPDRS motor exam/56 & $25.70 \pm 3.46$ & $22.60 \pm 4.47$ & $24.30 \pm 4.46$ & 1.34 & $0.22^{*}$ \\
LEDD, mg/day & $600 \pm 174.08$ & $600 \pm 174.08$ & $625 \pm 176.72$ & 0.06 & $0.93^{*}$ \\
\hline
\end{tabular}

Data in the table are presented as Mean \pm SD.

Abbreviations: CDTTG, Cognitive Dual-Task Training Group; MDTTG, Motor Dual-Task Training Group; STCG, Single Task Control Group; H\&Y, Hoehn \& Yahr stage; BMI, Body Mass Index; MMSE, Mini-Mental State Examination; UPDRS motor exam, Unified Parkinson's Disease Rating Scale motor exam; LEDD, Levodopa Equivalent Daily Dose.

* There is not any significant difference between clinical and demographic variables at baseline $(p>0.05)$.

Table 2. Estimated values and 95\% Confidence Intervals for Variables Before and After Intervention and After 1 Month Follow-up

\begin{tabular}{|c|c|c|c|c|c|c|c|c|c|}
\hline & \multicolumn{3}{|c|}{ Pre-intervention } & \multicolumn{3}{|c|}{ Post-intervention } & \multicolumn{3}{|c|}{1 month Follow-up } \\
\hline & CDTTG & MDTTG & STTCG & CDTTG & MDTTG & STTCG & CDTTG & MDTTG & STTCG \\
\hline TUG(s) & $\begin{array}{c}13.92(13.61 \\
-14.23)\end{array}$ & $\begin{array}{c}13.99(13.69 \\
-14.30)\end{array}$ & $\begin{array}{c}13.82(13.51 \\
-14.12)\end{array}$ & $\begin{array}{c}12.28(12.01 \\
-12.55)\end{array}$ & $\begin{array}{c}12.64(12.37 \\
-12.91)\end{array}$ & $\begin{array}{c}13.03(12.76 \\
-13.30)\end{array}$ & $\begin{array}{c}12.67(12.37 \\
-12.98)\end{array}$ & $\begin{array}{c}13.39(13.09 \\
-13.70)\end{array}$ & $\begin{array}{c}13.43(13.12 \\
-13.73)\end{array}$ \\
\hline $\begin{array}{l}\text { PDQ- } \\
39\end{array}$ & $\begin{array}{c}51.14(47.78 \\
-54.51)\end{array}$ & $\begin{array}{c}50.50(47.14 \\
-53.87)\end{array}$ & $\begin{array}{c}50.47(47.06 \\
-53.78)\end{array}$ & $\begin{array}{c}41.59(39.00 \\
-44.19)\end{array}$ & $\begin{array}{c}41.40(38.81 \\
-43.99)\end{array}$ & $\begin{array}{c}43.99(41.39 \\
-46.58)\end{array}$ & $\begin{array}{c}44.30(41.60 \\
-46.99)\end{array}$ & $\begin{array}{c}44.59(41.89 \\
-47.29)\end{array}$ & $\begin{array}{c}47.02(44.33 \\
-49.72)\end{array}$ \\
\hline TWT( & $\begin{array}{c}75.91(71.39 \\
-80.44)\end{array}$ & $\begin{array}{c}75.87(71.3 \\
5-80.40)\end{array}$ & $\begin{array}{c}72.45(67.93 \\
-76.97)\end{array}$ & $\begin{array}{c}65.86(62.0 \\
1-69.72)\end{array}$ & $\begin{array}{c}66.57(62.71 \\
-70.43)\end{array}$ & $\begin{array}{c}66.26(62.4 \\
1-70.12)\end{array}$ & $\begin{array}{c}70.11(65.97 \\
-74.25)\end{array}$ & $\begin{array}{c}70.80(66.66 \\
-74.94)\end{array}$ & $\begin{array}{c}70.04(65.90 \\
-74.80)\end{array}$ \\
\hline FES-I & $\begin{array}{c}34.90(31.03 \\
-38.76)\end{array}$ & $\begin{array}{c}31.20(27.33 \\
-35.06)\end{array}$ & $\begin{array}{c}28.90(25.03 \\
-32.76)\end{array}$ & $\begin{array}{c}26.40(23.45 \\
-29.34)\end{array}$ & $\begin{array}{c}23.20(20.2 \\
5-26.14)\end{array}$ & $\begin{array}{c}22.80(19.85 \\
-25.74)\end{array}$ & $\begin{array}{c}28.00(24.73 \\
-31.26)\end{array}$ & $\begin{array}{c}26.10(22.83 \\
-29.36)\end{array}$ & $\begin{array}{c}25.50(22.23 \\
-28.76)\end{array}$ \\
\hline
\end{tabular}

Abbreviations: CDTTG: Cognitive Dual-Task Training Group; MDTTG: Motor Dual-Task Training Group; STCG: Single Task Control Group.

Table 3. The Results of Within-Subjects and Between-Subjects Repeated Measure ANOVA for Variables

\begin{tabular}{|lcccccccccc|}
\hline & SS & DF & MS & F & p & SS & DF & MS & F & p \\
Within-Subjects & & & TUG (s) & & & & & PDQ - 39 & & \\
$\quad$ Factor 1 & 24.10 & 2 & 12.05 & 530.54 & $0.001^{*}$ & 1078.06 & 1.23 & 873.53 & 242.89 & $0.001^{*}$ \\
$\quad$ Factor 1* Group & 2.89 & 4 & 0.72 & 32.12 & $0.001^{*}$ & 40.64 & 2.46 & 16.64 & 4.57 & $0.001^{*}$ \\
$\quad$ Error & 1.2 & 54 & 0.02 & - & - & 119.83 & 33.32 & 3.59 & - & - \\
Between-Subjects & & & & & & & & & & \\
$\quad$ Group & 3.76 & 2 & 1.88 & 3.26 & 0.06 & 48.85 & 2 & 24.42 & 0.43 & 0.64 \\
$\quad$ Error & 15.54 & 27 & 0.57 & - & - & 1503.73 & 27 & 55.69 & - & - \\
Within-Subjects & & & TWT(s) & & & & & FES-I & & \\
$\quad$ Factor 1 & 1087.47 & 1.53 & 707.33 & 496.76 & $0.001 *$ & 888.62 & 1.58 & 561.13 & 319.90 & $0.001^{*}$ \\
$\quad$ Factor 1* Group & 49.69 & 3.07 & 16.16 & 11.35 & $0.001 *$ & 34.37 & 3.16 & 10.58 & 6.8 & $0.001^{*}$ \\
$\quad$ Error & 59.10 & 41.51 & 1.42 & - & - & 75.00 & 42.75 & 1.75 & - & - \\
Between-Subjects & & & & & & & & & & \\
$\quad$ Group & 35.29 & 2 & 17.64 & 0.14 & 0.86 & 260.82 & 2 & 130.41 & 1.65 & 0.20 \\
$\quad$ Error & 3306.76 & 27 & 122.47 & - & - & 2123.40 & 27 & 78.64 & - & - \\
\hline
\end{tabular}

Abbreviations: SS, Sum of Square; DF, Degree of Freedom; MS, Mean Square, CDTTG: Cognitive Dual-Task Training group; MDTTG: Motor Dual-Task Training Group; STCG: Single Task Control Group.

$*$ Significant effects are marked $(p<0.05)$ 
Table 4. Post Hoc Within-Group Comparisons: Pre-Intervention versus Post-Intervention, Pre-Intervention versus Follow-up, Post-

\begin{tabular}{|c|c|c|c|c|c|c|}
\hline \multicolumn{7}{|c|}{ Intervention versus Follow-up } \\
\hline & \multirow{2}{*}{\multicolumn{3}{|c|}{ TUG (s) }} & MD & $\mathbf{p}$ & ES \\
\hline & & & & \multicolumn{3}{|c|}{ PDQ-39 } \\
\hline \multicolumn{7}{|c|}{ Pre and post-intervention } \\
\hline CDTTG & 1.64 & $0.001 *$ & 9.64 & 9.55 & $0.001 *$ & 2.80 \\
\hline MDTTG & 1.35 & $0.001 *$ & 2.21 & 9.10 & $0.001 *$ & 1.53 \\
\hline STTCG & 0.78 & $0.001 *$ & 1.58 & 6.43 & $0.001 *$ & 1.11 \\
\hline \multicolumn{7}{|c|}{ Pre-intervention and Follow-up } \\
\hline CDTTG & 1.24 & $0.001 *$ & 7.35 & 6.84 & $0.001 *$ & 2.00 \\
\hline MDTTG & 0.60 & $0.001 *$ & 0.98 & 5.19 & $0.001 *$ & 0.99 \\
\hline STTCG & 0.39 & $0.001 *$ & 0.78 & 3.39 & $0.001 *$ & 0.58 \\
\hline \multicolumn{7}{|c|}{ Post-intervention and Follow-up } \\
\hline CDTTG & -0.39 & $0.001 *$ & 1.56 & -2.70 & $0.001 *$ & 1.38 \\
\hline MDTTG & -0.78 & $0.001 *$ & 1.41 & -3.18 & $0.001 *$ & 0.83 \\
\hline STTCG & -0.39 & $0.001 *$ & 0.97 & -3.03 & $0.001 *$ & 0.55 \\
\hline & \multicolumn{3}{|c|}{ TWT(s) } & \multicolumn{3}{|c|}{ FES-I } \\
\hline \multicolumn{7}{|c|}{ Pre and post-intervention } \\
\hline CDTTG & 10.04 & $0.001 *$ & 1.88 & 8.50 & $0.001 *$ & 2.00 \\
\hline MDTTG & 9.30 & $0.001 *$ & 1.19 & 8.00 & $0.001 *$ & 1.06 \\
\hline STTCG & 6.18 & $0.001 *$ & 0.82 & 6.10 & $0.001 *$ & 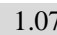 \\
\hline \multicolumn{7}{|c|}{ Pre-intervention and Follow-up } \\
\hline CDTTG & 5.80 & $0.001 *$ & 1.08 & 6.90 & $0.001 *$ & 1.62 \\
\hline MDTTG & 5.07 & $0.001 *$ & 0.74 & 5.10 & $0.001 *$ & 0.49 \\
\hline STTCG & 2.40 & $0.001 *$ & 0.32 & 3.40 & $0.001 *$ & 0.59 \\
\hline \multicolumn{7}{|c|}{ Post-intervention and Follow-up } \\
\hline CDTTG & -4.24 & $0.001 *$ & 1.02 & -1.60 & $0.001 *$ & 0.41 \\
\hline MDTTG & -4.23 & $0.001 *$ & 0.63 & -2.90 & $0.001 *$ & 0.52 \\
\hline STTCG & -3.78 & $0.001 *$ & 0.56 & -2.70 & $0.001 *$ & 0.67 \\
\hline
\end{tabular}

*Significant Effects are marked $(p<0.05)$

The Effect of Dual-Task and Single Task Training on Outcome Measures. In Table 2, estimated values and $95 \%$ confidence intervals for variables before and after the intervention and after one month follow-up are shown. According to Table 3, no interaction effects between Time and Group were found for TUG, PDQ-39, TWT, and FES-I which indicates that all training protocols had similar effects $(\mathrm{p}>0.05)$.

The main effects for the time were significant for TUG in within-subject comparisons in three groups $(\mathrm{F}=530.54, \mathrm{p}<0.001)($ Table 3$)$. Post hoc within-group analysis showed significant decreases in time of TUG test after treatment and after one-month follow-up in three groups $(\mathrm{p}<$ 0.05) (Table 4).

For PDQ - 39, the main effects were significant in within-subject comparisons in three groups $(\mathrm{F}$ $=242.89, \mathrm{p}<0.001$ ) (Table 3). Post hoc withingroup analysis showed a significant decrease in score of PDQ-39 after treatment and after onemonth follow-up in three groups $(\mathrm{p}<0.05)$ (Table 4). The main effects were significant for TWT in within-subject comparisons in three groups $(\mathrm{F}=$ 496.76, p < 0.001) (Table 3). Post hoc withingroup analysis showed significant decreases in time of TWT after treatment and after one-month follow-up in three groups $(\mathrm{p}<0.05)$ (Table 4).

For the last outcome measure, the main effects were significant in within-subject comparisons in three groups, too $(\mathrm{F}=319.90, \mathrm{p}<0.001)$ (Table $3)$. Post hoc within-group analysis showed significant decreases in the score of FES-I after treatment and after one-month follow-up in three groups $(\mathrm{p}<0.05)$ (Table 4) (Figures 1-4).

\section{DISCUSSION}

In this study, we compared the efficacy of two dual-task training programs and a single task training program on improvement of balance, quality of life, and fear of falls in people with idiopathic Parkinson's disease. The results of this study indicate that cognitive/motor dual-task training and single-task training programs were effective in improving balance, quality of life, and fear of falls. These effects were obvious not only in the single-task training group but also in the dual-task training groups, and were maintained for one month after training. Patients with PD suffer from loss of automaticity in movements. Thus, it is not surprising that several studies revealed balance disorders in patients with PD under DT conditions compared with healthy agematched control groups (26). Currently, according to the evidence-based rehabilitation guidelines in PD it is better to avoid DT situations and divide complex tasks in easier subcomponents (27). But in recent years, European guideline provides a different opinion, stating that in Hoehn and Yahr stages 2 and 3 DT training may be safe and effective (25). 


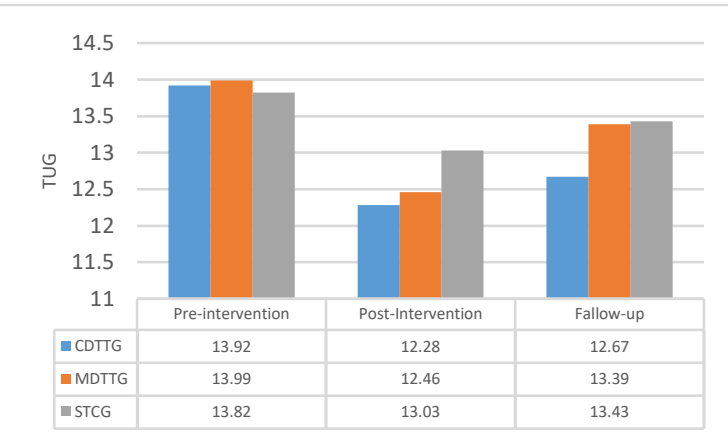

Figure 1. TUG changes. Bar represents TUG changes in three groups before and after the intervention and after one-month follow-up. Abbreviations: CDTTG: Cognitive Dual-Task Training group, MDTTG: Motor Dual-Task Training Group, STCG: Single Task Control Group

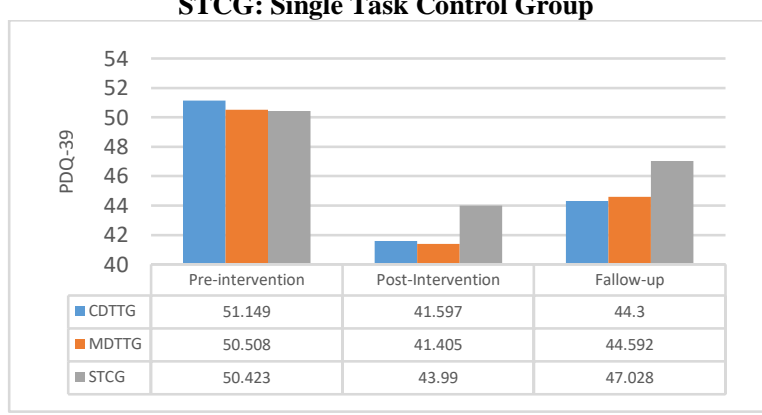

Figure 2. PDQ-39 Changes. The bar represents PDQ-39 Changes in Three Groups Before and After Intervention and After One Month Follow-up. Abbreviations: CDTTG: Cognitive Dual-Task Training group, MDTTG: Motor DualTask Training Group, STCG: Single Task Control Group

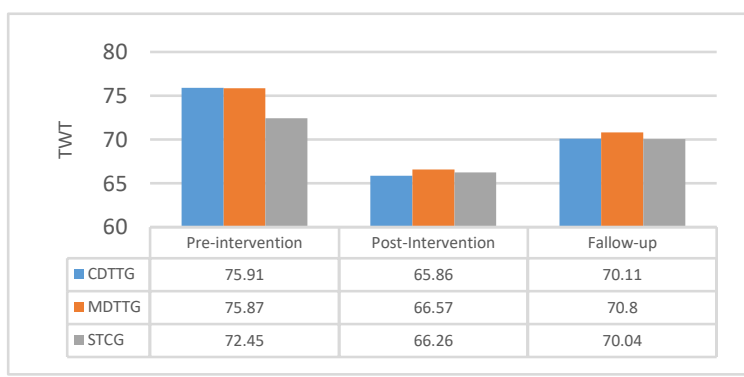

Figure 3. TWT Changes. The bar represents TWT Changes in Three Groups Before and After Intervention and After One Month Follow-up. Abbreviations: CDTTG: Cognitive DualTask Training group, MDTTG: Motor Dual-Task Training Group, STCG: Single Task Control Group

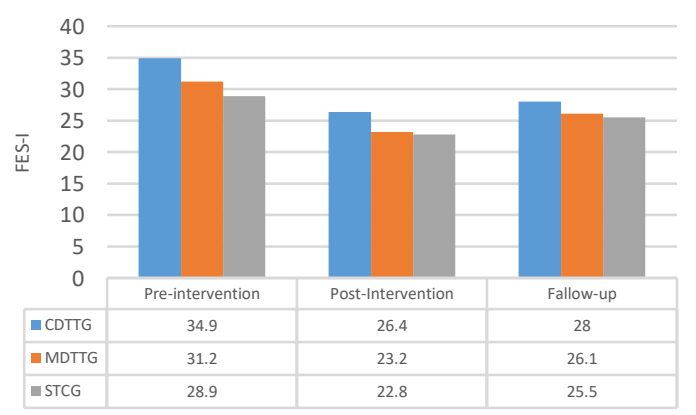

Figure 4. FES-I changes. Bar represents FES-I Changes in Three Groups Before and After Intervention and After One Month Follow-up. Abbreviations: CDTTG: Cognitive DualTask Training group, MDTTG: Motor Dual-Task Training Group, STCG: Single Task Control Group
Most of the studies targeted the effect of dualtask training on improvement of gait parameters. Although gait analysis is very important in PD, the other issues like balance and quality of life are valuable. The findings of this study showed that cognitive/motor dual-task training could make improvements in TUG and this improvement remained even after one-month Follow-up. In agreement with our findings, Romenets et al. reported significant improvements in TUG and DT-TUG performance in comparison to the control group after 12 weeks Tango dancing (28). In spite of different type of DT training the effect of training on TUG were similar in these studies. De Freitas et al in a systematic review showed that dual-task training can make improvement of balance and performance of executive functions. As stated in their study DT training can cause better result in TUG and MiniBESTest (29). In the current study, we assessed the effect of DT training on a functional test called TWT. This test is designed by Yamada and Ichihashi in 2010 and it is an improved version of Trail-Marking Test (TMT) and more difficult than it. The TWT includes walking from numbered flags in ascending or descending orders. Cognitive functions like visual search function and shortterm memory simultaneous with motor functions like locomotion and turning are needed to successfully perform the TWT (22). The results of this study indicated that cognitive/motor dualtask training could cause faster TWT after training and after one month Follow-up. To the best of our knowledge, there is not any study to show the effect of DT training on TWT in PD. Any other way, it is confirmed that TWT and TUG can predict falling in community-dwelling elderly individuals (18). In such a way, faster TWT and TUG time are related to the decrease in fall risk. In the present study, we could show this effect of DT training on fear of falls, variously. According to the results of this study, cognitive/motor DT training can decrease the fear of falls using the FES-I questionnaire. The results of previous studies support this finding. Sahu et al. evaluated the effect of DT training on fear of fall (FOF) using the Tinetti Fall Efficacy Scale and they could show significant improvement in FOF after training (29). Strouwen et al. compared the efficacy of integrated dual-task training (IDT) and continuous dual-task training (CDT) on gait parameters and risk of fall in patients with PD. Although they could show a decrease in fall risk 
in both groups, that effect was not statistically significant (11). A dual-task acutely directs the performer's attention toward an external source of attention, while performing a primary task. As stated in constrained action hypothesis, this attentional change might allow motor systems to function automatically, resulting in more effective performance (29)As mentioned above, DT training could improve balance and decrease fear of falls along these lines it can make better quality of life in patients and we could show it, too. Lofgren et al. in a secondary analysis from a randomized trial, stated that integrated single and DT training could improve the automaticity of cognitive processing during walking, thereby improve the quality of life in PD patients (29). In contrast to our hypothesis, no significant difference between groups was found. It seems that is why the lack of an actual control group without any intervention. As reported by studies of motor learning the effect of DT training will transfer not only to the DT performances but also to the single task performances (12). That could be another reason for the lack of any significant differences between STCG and DT training groups in this study. A limitation of this study is that $\mathrm{PD}$ is a progressive disease. Although the duration of the training protocol was just ten weeks, PD progression might have influenced the outcome measures in the current research.
Another limitation in this study is the small sample size which limits the ability to generalize its results to a wider population. Further studies with a larger sample size are needed.

\section{CONCLUSIONS}

In conclusion, single task and motor/cognitive DT training were found to be moderately effective in improvement of balance, quality of life, and decrease of fear of falls in people with PD. The effects retained for one month that is indicative of motor learning capacity in PD. In opposition to current thinking, DT training was not as hazardous as fear of falls decreased. Thus, these training protocols should be included among the rehabilitative approaches that physiotherapists use in their clinical practice.

\section{APPLICABLE REMARKS}

DT training should be included in the rehabilitation program by physiotherapists in their clinical practice.

\section{ACKNOWLEDGMENTS}

This study is part of a doctoral thesis by the first author of the article, and it was conducted by the Faculty of Physical Education and Sport Science of the University of Guilan. The authors would like to thank the personnel of The University of Guilan for their dedicated supports. They would also like to thank all the patients who took part in this study.

\section{REFERENCES}

1. Schapira AH, Olanow CW. Neuroprotection in Parkinson disease: mysteries, myths, and misconceptions. JAMA. 2004;291(3):358-364. doi: 10.1001/jama.291.3.358 pmid: 14734599

2. Dorsey ER, Constantinescu R, Thompson JP, Biglan KM, Holloway RG, Kieburtz K, et al. Projected number of people with Parkinson disease in the most populous nations, 2005 through 2030. Neurology. 2007;68(5):384-386. doi: 10.1212/01.wnl.0000247740.47667.03 pmid: 17082464

3. Santos LAA, Campos C, Bento T, Lattari E, Nardi AE, Rocha NBF, et al. Effects of dual-task interventions on gait performance of patients with Parkinson's Disease: A systematic review. Med Expr. 2016;3(4). doi: 10.5935/MedicalExpress.2016.04.01

4. Peterson DS, King LA, Cohen RG, Horak FB. Cognitive Contributions to Freezing of Gait in Parkinson Disease: Implications for Physical Rehabilitation. Phys Ther. 2016;96(5):659-670. doi: 10.2522/ptj.20140603 pmid: 26381808

5. Brauer SG, Woollacott MH, Lamont R, Clewett S, O'Sullivan J, Silburn P, et al. Single and dual task gait training in people with Parkinson's disease: a protocol for a randomised controlled trial. BMC Neurol. 2011;11:90. doi: 10.1186/1471-2377-11-90 pmid: 21791117

6. Ashburn A, Stack E, Ballinger C, Fazakarley L, Fitton C. The circumstances of falls among people with Parkinson's disease and the use of Falls Diaries to facilitate reporting. Disabil Rehabil. 2008;30(16):1205-1212. doi: 10.1080/09638280701828930 pmid: 18608387

7. Soh SE, Morris ME, McGinley JL. Determinants of health-related quality of life in Parkinson's disease: a systematic review. Parkinsonism Relat Disord. 2011;17(1):1-9. doi: 10.1016/j.parkreldis.2010.08.012 pmid: 20833572 
8. Heinzel S, Maechtel M, Hasmann SE, Hobert MA, Heger T, Berg D, et al. Motor dual-tasking deficits predict falls in Parkinson's disease: A prospective study. Parkinsonism Relat Disord. 2016;26:73-77. doi: 10.1016/j.parkreldis.2016.03.007 pmid: 26997654

9. Spildooren J, Vercruysse S, Desloovere K, Vandenberghe W, Kerckhofs E, Nieuwboer A. Freezing of gait in Parkinson's disease: the impact of dual-tasking and turning. Mov Disord. 2010;25(15):2563-2570. doi: 10.1002/mds.23327 pmid: 20632376

10.Fuller RL, Van Winkle EP, Anderson KE, Gruber-Baldini AL, Hill T, Zampieri C, et al. Dual task performance in Parkinson's disease: a sensitive predictor of impairment and disability. Parkinsonism Relat Disord. 2013;19(3):325-328. doi: 10.1016/j.parkreldis.2012.11.011 pmid: 23265679

11. Strouwen C, Molenaar E, Munks L, Keus SHJ, Zijlmans JCM, Vandenberghe W, et al. Training dual tasks together or apart in Parkinson's disease: Results from the DUALITY trial. Mov Disord. 2017;32(8):1201-1210. doi: 10.1002/mds.27014 pmid: 28440888

12. Kelly VE, Eusterbrock AJ, Shumway-Cook A. The effects of instructions on dual-task walking and cognitive task performance in people with Parkinson's disease. Parkinsons Dis. 2012;2012:671261. doi: 10.1155/2012/671261 pmid: 23326758

13. Cunningham JB, McCrum-Gardner E. Power, effect and sample size using GPower: practical issues for researchers and members of research ethics committees. Eviden Bas Midwifery. 2007;5(4):132-137.

14. van den Heuvel MR, Kwakkel G, Beek PJ, Berendse HW, Daffertshofer A, van Wegen EE. Effects of augmented visual feedback during balance training in Parkinson's disease: a pilot randomized clinical trial. Parkinsonism Relat Disord. 2014;20(12):1352-1358. doi: 10.1016/j.parkreldis.2014.09.022 pmid: 25283070

15. Yang Y, Hao YL, Tian WJ, Gong L, Zhang K, Shi QG, et al. The effectiveness of Tai Chi for patients with Parkinson's disease: study protocol for a randomized controlled trial. Trials. 2015;16:111. doi: 10.1186/s13063-015-0639-8 pmid: 25873334

16. Nocera JR, Stegemoller EL, Malaty IA, Okun MS, Marsiske M, Hass CJ, et al. Using the Timed Up \& Go test in a clinical setting to predict falling in Parkinson's disease. Arch Phys Med Rehabil. 2013;94(7):1300-1305. doi: 10.1016/j.apmr.2013.02.020 pmid: 23473700

17. Ghaem H, BORHANI HA, Zeyghami B, Dehghan A. Validity and reliability of the Persian version of the Parkinson disease quality of life (PDQL) questionnaire. J Kerman univ med sci. 2010;17(1):49-58.

18.Zhang JL, Chan P. Reliability and validity of PDQ-39: a quality-of-life measure for patients with PD in China. Qual Life Res. 2012;21(7):1217-1221. doi: 10.1007/s11136-011-0026-1 pmid: 21983714

19. Dehghan A, Ghaem H, Borhani-Haghighi A, Safari-Faramani R, Moosazadeh M, Gholami A. Evaluation of Reliability and Validity of PDQ-39: Questionnaire in Iranian Patients With Parkinson's Disease. Zahedan J Res Med Sci. 2016;18(3). doi: 10.17795/zjrms-6245

20. Yamada M, Ichihashi N. Predicting the probability of falls in community-dwelling elderly individuals using the trail-walking test. Environ Health Prev Med. 2010;15(6):386-391. doi: 10.1007/s12199-0100154-1 pmid: 21432571

21. Marques Vieira CMA, Sousa LMM, Severino S, Sousa L, Caldeira S. Cross-cultural validation of the falls efficacy scale international in elderly: Systematic literature review. J Clin Gerontol Geriatr. 2016;7(3):72-76. doi: 10.1016/j.jcgg.2015.12.002

22. Sacheli MA, Murray DK, Vafai N, Cherkasova MV, Dinelle K, Shahinfard E, et al. Habitual exercisers versus sedentary subjects with Parkinson's Disease: Multimodal PET and fMRI study. Mov Disord. 2018;33(12):1945-1950. doi: 10.1002/mds. 27498 pmid: 30376184

23.Canning CG, Ada L, Woodhouse E. Multiple-task walking training in people with mild to moderate Parkinson's disease: a pilot study. Clin Rehabil. 2008;22(3):226-233. doi: 10.1177/0269215507082341 pmid: 18285432

24. Keus SH, Bloem BR, Hendriks EJ, Bredero-Cohen AB, Munneke M, Practice Recommendations Development G. Evidence-based analysis of physical therapy in Parkinson's disease with recommendations for practice and research. Mov Disord. 2007;22(4):451-460; quiz 600. doi: 10.1002/mds. 21244 pmid: 17133526

25.Rios Romenets S, Anang J, Fereshtehnejad SM, Pelletier A, Postuma R. Tango for treatment of motor and non-motor manifestations in Parkinson's disease: a randomized control study. Complement Ther Med. 2015;23(2):175-184. doi: 10.1016/j.ctim.2015.01.015 pmid: 25847555 
26. De Freitas Tb Ms PT, Leite PB, Dona F PhD PT, Pompeu Je PhD PT, Swarowsky A PhD PT, TorrianiPasin C PhD PT. The effects of dual task gait and balance training in Parkinson's disease: a systematic review. Physiother Theory Pract. 2018:1-9. doi: 10.1080/09593985.2018.1551455 pmid: 30501424

27. Sahu S, Jali P, Srivastava A. Dual task training, fall, and functional independence in patients with Parkinson's disease: A longitudinal study. The Indian Journal of Occupational Therapy. 2018;50(4):134.

28. Ghai S, Ghai I, Effenberg AO. Effects of dual tasks and dual-task training on postural stability: a systematic review and meta-analysis. Clin Interv Aging. 2017;12:557-577. doi: 10.2147/CIA.S125201 pmid: 28356727

29.Lofgren N, Conradsson D, Rennie L, Moe-Nilssen R, Franzen E. The effects of integrated single- and dual-task training on automaticity and attention allocation in Parkinson's disease: A secondary analysis from a randomized trial. Neuropsychology. 2019;33(2):147-156. doi: 10.1037/neu0000496 pmid: 30407031 